\title{
Distributed Deployment Strategies for Improved Coverage in a Network of Mobile Sensors with Prioritized Sensing Field
}

\author{
Hamid Mahboubi, Student Member, IEEE, Jalal Habibi, Member, IEEE, \\ Amir G. Aghdam, Senior Member, IEEE, and Kamran Sayrafian-Pour, Senior Member, IEEE
}

\begin{abstract}
Efficient deployment strategies are proposed for a mobile sensor network, where the coverage priority of different points in the field is specified by a priority function. The multiplicatively weighted Voronoi (MW-Voronoi) diagram is utilized to find the coverage holes of the network for the case where the sensing ranges of different sensors are not the same. Under the proposed strategies, each sensor detects coverage holes within its MW-Voronoi region, and then moves in a proper direction to reduce their size. Since the coverage priority of the field is not uniform, the target location of each sensor is determined based on the weights of the vertices or the points inside the corresponding MW-Voronoi region. Simulations validate the theoretical results.
\end{abstract}

Index Terms-Coverage, distributed deployment algorithm, mobile sensors, prioritized sensing field, wireless sensor networks,

\section{INTRODUCTION}

W IRELESS sensor networks have drawn much attention recently due to their various civilian and military applications. Recent advances in microelectromechanical systems (MEMS) technology have made it possible to fabricate small energy-efficient mobile sensors. Some of the emerging applications of cooperative mobile sensor networks include health-monitoring systems, space exploration and environmental assessment, to name only a few [1], [2], [3]. Over the past two decades, researchers in different disciplines of engineering and computer science have made significant contributions in this area by deriving accurate models for such systems and developing cost-effective deployment algorithms for different purposes [4], [5], [6].

In the design of a practical sensor deployment strategy, some important constraints need to be taken into account. Such constraints include limited sensing and communication ranges of sensors, limited energy of sensors, and limited information exchange between them [7], [8], [9]. Furthermore, the initial location of the sensors in the field is not known a priori in many practical applications [10]. The problem of network coverage by a group of mobile sensors following a prescribed

H. Mahboubi, J. Habibi and A. G. Aghdam are with the Department of Electrical \& Computer Engineering, Concordia University, 1455 de Maisonneuve Blvd. W., EV012.179, Montréal, Québec H3G 1M8 Canada.

E-mail: \{h_mahbo, jalal, aghdam\}@ece.concordia.ca

K. Sayrafian-Pour is with the National Institute of Standards and Technology (NIST), 100 Bureau Drive, Stop 8920 Gaithersburg, MD 20899 USA. E-mail: \{ksayrafian\}@nist.gov

Copyright (c) 2012 IEEE. Personal use of this material is permitted. However, permission to use this material for any other purposes must be obtained from the IEEE by sending a request to pubs-permissions@ieee.org.

This work has been supported by the National Institute of Standards and Technology (NIST), under grant 70NANB8H8146. trajectory is investigated in [11]. A multi-objective optimal sensor deployment and power assignment algorithm is proposed in [12], where the problem is decomposed into a number of scalar single-objective subproblems which are to be solved simultaneously. In [8], [9], multiplicatively weighted Voronoi (MW-Voronoi) diagram is used to partition the sensing field in a network of mobile sensors with nonidentical sensing ranges. Different deployment strategies are subsequently introduced to improve sensing coverage of such networks. Vector-based and Voronoi-based algorithms are proposed in [7] to move the sensors in the field in such a way that network coverage increases. In [13], basic protocols and virtual movement protocols are introduced for sensor deployment to increase network coverage. Gradient-descent coverage algorithms are presented in [14], which are distributed in the sense of the Delaunay graph. In [15], coordination algorithms are provided for sensor deployment and coverage, where a class of aggregate objective functions is also considered based on the geometry of the Voronoi partitions and proximity graphs.

In most of the above-mentioned sensor deployment algorithms, it is assumed that the coverage priority for different points in the network is uniform (i.e., the importance of coverage of every point in the network is the same). While this is a realistic assumption in many real-world problems, sometimes certain areas in the network have higher priority as far as coverage is concerned, due, for example, to safety considerations. The sensor deployment problem in a nonuniform field is considered in [15], [16], [17]. However, due to the computational complexity of the corresponding techniques, they may not be suitable for the case when the processing capability of the sensors in the network is limited. To the best of the authors' knowledge, there is no result in the literature so far concerning coverage of a prioritized field with nonidentical sensors.

In this work, new distributed deployment strategies are introduced to increase coverage in a network of mobile sensors with a prescribed priority function for the sensing field. To this end, a priority function is assumed to be given which specifies the coverage priority of different points in the sensing area. The MW-Voronoi diagram is used to partition the sensing field [18], [19], [20]. This partitioning is then used to discover coverage holes in the network and relocate the sensors accordingly to minimize them, while taking into account the coverage priority of different points in the field. Three algorithms are developed: maximum weighted vertex (MWV), maximum weighted point (MWP), and maximum distance weight (MDW). The main idea behind the proposed algorithms 
is to move each sensor iteratively in such a way that its weighted coverage increases. Once each sensor's destination is computed, new local weighted coverage of the corresponding sensor in the previously constructed MW-Voronoi region is compared to its previous local weighted coverage. If the new local weighted coverage is larger than the previous one, the sensor moves to the new location; otherwise, it remains in its current position. If in an iteration the local weighted coverage by none of the sensors is improved by a certain amount, then the algorithm is terminated (to ensure a finite number of iterations).

The plan of the rest of the paper is as follows. Section II provides the required background for understanding the theoretical results. The problem is formulated in Section III, and some important assumptions and definitions are also given which will be used later to develop the main results. Section IV presents the main contributions of the paper, where new deployment algorithms are introduced. The proposed algorithms are compared in Section V, and finally concluding remarks are drawn in Section VI.

\section{BACKGROUND}

\section{A. Voronoi Diagram}

Let $\overline{\mathbf{S}}$ be a set of $n$ distinct nodes in the plane denoted by $S_{1}, S_{2}, \ldots, S_{n}$. It is desired to partition the plane into $n$ polygons such that:

- Each polygon contains only one node, and

- the nearest node to any point inside a polygon is the node assigned to that polygon.

The diagram obtained by the partitioning described above is called the Voronoi diagram [21]. To construct the Voronoi polygon associated with a node in the network, first the perpendicular bisector of every segment connecting the node to any of its neighbors is drawn. The smallest polygon (created by the above perpendicular bisectors) containing the node is, in fact, the Voronoi polygon of that node.

The Voronoi diagram is very useful for the analysis and design of sensor deployment techniques. Represent each sensor in the field as a node, and sketch the Voronoi polygons for all sensors as described above, to partition the entire target field (which is also assumed to be a polygon). From the characterization of the Voronoi diagram, assuming that all sensors have the same sensing capability, it is straightforward to show that if a sensor cannot cover a certain point in its corresponding polygon, other sensors in the network cannot cover it either. This means that in order to find the so-called "coverage holes" (the points that cannot be covered by the sensors), it would suffice to find the intersection of the Voronoi polygon of each sensor with the exterior of its local coverage area. However, as noted earlier, this fundamental statement is only true for the case when all sensors have the same sensing range. It can be easily shown that when the sensors are not identical in terms of sensing radius, a point which is not detected by the sensor located in the same polygon, may be detectable by the sensor corresponding to a neighboring polygon. Hence, when the sensing radii of different sensors are not the same, the conventional Voronoi diagram is not as useful for effective sensor deployment in the network. The partitioning described in the next subsection is used to remedy this problem.

\section{B. Multiplicatively Weighted Voronoi Diagram}

Let $\mathbf{S}$ be a set of $n$ distinct weighted nodes $\left(S_{1}, w_{1}\right),\left(S_{2}, w_{2}\right), \ldots,\left(S_{n}, w_{n}\right)$ in the plane, where $w_{i}>0$ is the weighting factor associated with $S_{i}$, for any $i \in \mathbf{n}:=\{1,2, \ldots, n\}$. Partition the plane into $n$ regions such that each region contains only one node, which is the nearest node, in the sense of weighted distance, to any point inside that region. The diagram obtained by the partitioning described above is called the multiplicatively weighted Voronoi diagram (MW-Voronoi diagram) [19]. Analogous to the conventional Voronoi diagram, the mathematical characterization of each region in this case is given by:

$$
\Pi_{i}=\left\{q \in \mathbb{R}^{2} \mid w_{j} d\left(q, S_{i}\right) \leq w_{i} d\left(q, S_{j}\right), \forall j \in \mathbf{n} \backslash\{i\}\right\}
$$

where $d\left(q, S_{i}\right)$ denotes the Euclidean distance between the point $q$ and the node $S_{i}$ in the 2D plane. According to (1), any point $q$ in the $i$-th MW-Voronoi region $\Pi_{i}$ has the following property:

$$
\frac{d\left(q, S_{i}\right)}{d\left(q, S_{j}\right)} \leq \frac{w_{i}}{w_{j}}, \quad \forall i \in \mathbf{n}, \forall j \in \mathbf{n} \backslash\{i\}
$$

Definition 1. The nodes $S_{i}$ and $S_{j}$ are called neighbors if $\Pi_{i} \cap$ $\Pi_{j} \neq \varnothing$ (note that the intersection of two neighboring regions includes the boundary points only). The set of all neighbors of $S_{i}$ is denoted by $\mathbf{N}_{i}$, i.e.:

$$
\mathbf{N}_{i}=\left\{S_{j} \in \mathbf{S} \mid \Pi_{i} \cap \Pi_{j} \neq \varnothing\right\}
$$

Definition 2. Given a positive real constant $k$, the Apollonian circle of a segment $A B$, denoted by $\Omega_{A B, k}$, is the locus of all points $E$ such that $\frac{A E}{B E}=k$ [22].

To construct the $i$-th MW-Voronoi region, first the Apollonian circles $\Omega_{S_{i} S_{j}, \frac{w_{i}}{w_{j}}}$ are found for all $S_{j} \in \mathbf{N}_{i}$. The smallest closed region (created by the above circles) containing the $i$-th node is, in fact, the $i$-th MW-Voronoi region (e.g., see Fig. 1). An example of an MW-Voronoi diagram with 15 weighted nodes is sketched in Fig. 2.

The MW-Voronoi diagram is the main tool for developing sensor deployment algorithms in this paper. Denote each sensor in the field as a node with a weight equal to its sensing radius, and sketch the MW-Voronoi region for every sensor. The following definition proves convenient in the development of sensor deployment algorithms in this paper.

Definition 3. Consider a sensor $S_{i}$ with the sensing radius $r_{i}$ and the corresponding MW-Voronoi region $\Pi_{i}, i \in \mathbf{n}$, and let $q$ be an arbitrary point inside $\Pi_{i}$. The intersection of the region $\Pi_{i}$ and a circle of radius $r_{i}$ centered at $q$ is referred to as the coverage area w.r.t. q. The coverage area w.r.t. the location of the sensor $S_{i}$ is called the local coverage area of that sensor. 


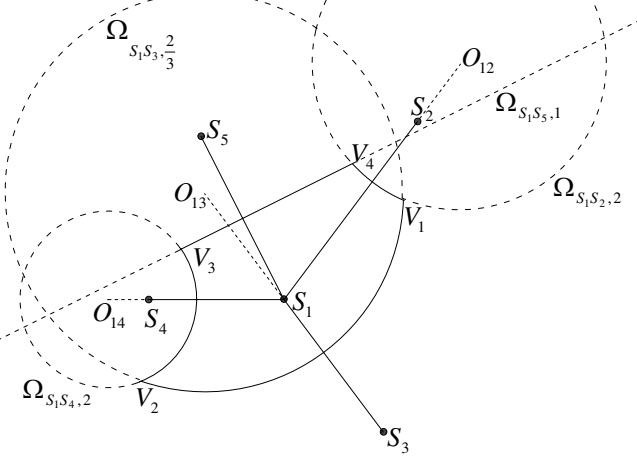

Fig. 1: The MW-Voronoi region for a node $S_{1}$ with four neighbors $S_{2}, \ldots, S_{5}$ [8].

From the characterization of the MW-Voronoi regions provided in (1), it is straightforward to show that if a sensor cannot detect a point in its corresponding region, no other sensor can detect it either. This means that in order to find the coverage holes, it would suffice to compare the MW-Voronoi region of each node with its local coverage area.

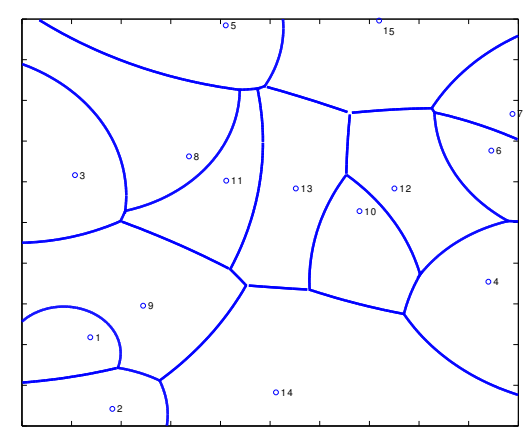

Fig. 2: An example of the MW-Voronoi diagram for a network of 15 weighted nodes.

\section{Problem Formulation}

Consider a group of $n$ mobile sensors, randomly distributed in the sensing field, and let the sensing radius of the $i$-th sensor be denoted by $r_{i}$ (note that the sensing radii of the sensors can be different). The coverage priority of different points in the field is assumed to be specified by a priority function $\varphi(q)$. In other words, the coverage importance of the point $q$ is more than that of point $p$ if $\varphi(q)>\varphi(p)$.

It is desired to move the sensors and place them in proper positions in the field using a distributed deployment strategy such that the more important points are covered as much as possible. In other words, the objective is to increase the weighted coverage area with limited information exchange between sensors.
Definition 4. The integral of the priority function over the MW-Voronoi region $\Pi_{i}$ is referred to as the $i$-th region weight, and is denoted by $\alpha_{\Pi_{i}}$, i.e.:

$$
\alpha_{\Pi_{i}}=\int_{\Pi_{i}} \varphi(q) d q
$$

Also, the integral of the priority function over the entire sensing field is referred to as the total field weight, and is denoted by $\alpha$. It is straightforward to show that $\alpha=\sum_{i=1}^{n} \alpha_{\Pi_{i}}$.

Definition 5. Consider the sensor $S_{i}$ with the sensing radius $r_{i}$ and the corresponding MW-Voronoi region $\Pi_{i}, i \in \mathbf{n}$, and let $x$ be an arbitrary point inside $\Pi_{i}$. The integral of the priority function over the $i$-th coverage area w.r.t. $x$ (see Definition 3) is referred to as the $i$-th weighted coverage w.r.t. $x$, and is mathematically characterized as:

$$
\beta_{\Pi_{i}}^{x}=\int_{\Pi_{i} \cap C\left(x, r_{i}\right)} \varphi(q) d q
$$

where $C\left(x, r_{i}\right)$ is a circle of radius $r_{i}$ centered at $x$. Also, the $i$-th weighted hole w.r.t. $x$ is denoted by $\theta_{\Pi_{i}}^{x}$, and is expressed as:

$$
\theta_{\Pi_{i}}^{x}=\alpha_{\Pi_{i}}-\beta_{\Pi_{i}}^{x}
$$

Inside an MW-Voronoi region, the weighted covered and uncovered areas w.r.t. the location $p_{i}$ of the sensor $S_{i}$ (i.e. $\beta_{\Pi_{i}}^{p_{i}}$ and $\left.\theta_{\Pi_{i}}^{p_{i}}\right)$ are called the $i$-th local weighted coverage and $i$-th local weighted hole of that sensor, respectively. Furthermore, the integral of the priority function over the covered area (noncovered area) in the field is referred to as the total weighted coverage (total weighted hole).

Assumption 1. In this paper, it is assumed that there is no obstacle in the field. Therefore, the sensors can move to any desired location using existing techniques such as the ones introduced in [23], [24].

Assumption 2. The sensors are capable of localizing themselves with sufficient accuracy in the field (using, for instance, the methods proposed in [25], [1]).

\section{Deployment PRotocols}

In this section, three distributed deployment algorithms are introduced for a mobile sensor network. The proposed deployment algorithms perform iteratively until a prespecified termination condition is satisfied. Each iteration in the proposed algorithms consists of four phases. In the first phase, every sensor broadcasts its location and sensing radius to other sensors, and constructs its MW-Voronoi region subsequently based on the information it receives from other sensors. Then in the second phase, each sensor uses the available information to compute its destination point in its MW-Voronoi region according to the specific deployment strategy. Once the new target location $\dot{p}_{i}$ is determined, the weighted coverage area w.r.t. this location (i.e. $\beta_{\Pi_{i}}^{\hat{p}_{i}}$ ) is obtained in the third phase. If this value is greater than the previous local weighted coverage area (i.e. $\beta_{\Pi_{i}}^{p_{i}}>\beta_{\Pi_{i}}^{p_{i}}$ ), then the sensor moves to the new des- 


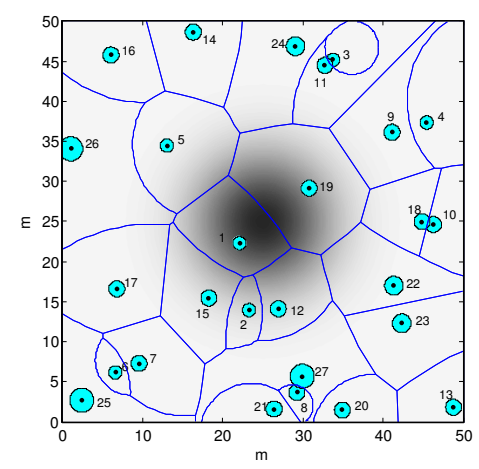

(a)

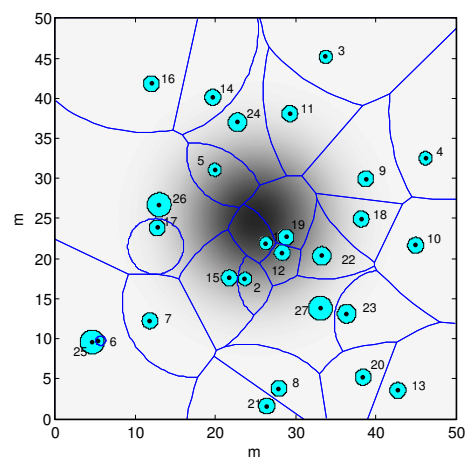

(b)

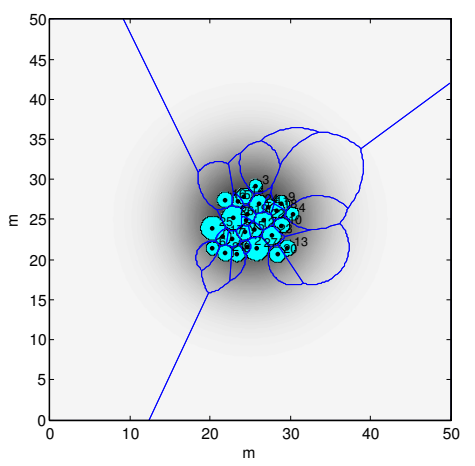

(c)

Fig. 3: Snapshots of the execution of the MWV strategy for a sensing field, where the coverage priority of different points in it is depicted by different gray levels (the white color represents the lowest priority and the black color the highest). (a) Initial coverage; (b) coverage after the first round, and (c) final coverage.

tination; otherwise, it remains in its current position. Finally, in the termination phase, if the local weighted coverage area by none of the sensors is improved by a certain amount, then the iteration stops. Note that the first, third and fourth phases described above are exactly the same. Thus, the algorithms introduced later in this section differ only in the second phase where the new location of each sensor is determined.

Assumption 3. It is implicitly assumed that a synchronization protocol (similar to one in [26]) is implemented to guarantee that all sensors start the first phase at the same time. Furthermore, the coverage rounds are assumed to be sufficiently long, so that all four phases described above can be completed in one round.

As noted above, one of the important characteristics of the sensor deployment strategies proposed in this section is that each sensor moves to its new destination point only if its weighted coverage w.r.t. the new location in the old MWVoronoi region increases. It is shown in the next theorem that the total weighted coverage under this type of deployment scheme increases, in general.

Theorem 1. Consider a set $\mathbf{S}$ of $n$ sensors in the plane, and let their positions be denoted by $\mathbf{P}=\left\{p_{1}, p_{2}, \ldots, p_{n}\right\}$ with the corresponding $M W$-Voronoi regions $\Pi_{1}, \Pi_{2}, \ldots, \Pi_{n}$. Assume the sensors move to new locations $\mathbf{P}=\left\{\dot{p}_{1}, \dot{p}_{2}, \ldots, \dot{p}_{n}\right\}$ with the corresponding $M W$-Voronoi regions $\Pi_{1}, \Pi_{2}, \ldots, \Pi_{n}$, and define the non-empty set $\mathbf{K} \subseteq \mathbf{n}$ in such a way that $p_{i} \neq p_{i}$ if and only if $i \in \mathbf{K}$. If the $i$-th weighted coverage w.r.t. $p_{i}$ in the previously constructed $M W$-Voronoi region $\Pi_{i}$ is greater than the $i$-th local weighted coverage in $\Pi_{i}$ (i.e., $\beta_{\Pi_{i}}^{p_{i}}>\beta_{\Pi_{i}}^{p_{i}}$ ) for all $i \in \mathbf{K}$, then the total weighted coverage in the network increases.

Remark 1. It is important to note that the statement of Theorem 1 is not trivial at all. To clarify this issue, notice that each sensor finds a new point in its MW-Voronoi region with the objective of increasing its local coverage. Since the geometry of the regions will change based on the new positions of the sensors, the achieved local coverage of each sensor in the new region is not necessarily more than the local coverage in the previous MW-Voronoi region. However, the subtle point of the theorem is that the total coverage would be monotonically increasing using this type of strategy.

Proof of Theorem 1: Suppose the total weighted hole of the field when the sensors are located in $\mathbf{P}$ and $\hat{\mathbf{P}}$ are denoted by $\theta$ and $\dot{\theta}$, respectively. From the characterization of the MWVoronoi diagram, one can conclude that:

$$
\theta=\sum_{i=1}^{n} \theta_{\Pi_{i}}^{p_{i}}
$$

It is straightforward to show that by increasing the weighted coverage in $\Pi_{i}, i \in \mathbf{K}$, the corresponding weighted hole will decrease. Since it is assumed that the $i$-th weighted coverage w.r.t. $\dot{p}_{i}$ is greater than the $i$-th local weighted coverage for any $i \in \mathbf{K}$, one can conclude that:

$$
\theta_{\Pi_{i}}^{\dot{p}_{i}}<\theta_{\Pi_{i}}^{p_{i}}, \quad \forall i \in \mathbf{K}
$$

On the other hand, some of the points in $\theta_{\Pi_{i}}^{p_{i}}$ might also be covered by another sensor located at $\dot{p}_{j}$, for some $j \in \mathbf{n} \backslash\{i\}$. Thus:

$$
\dot{\theta} \leq \sum_{i=1}^{n} \theta_{\Pi_{i}}^{\dot{p}_{i}}
$$

From the last two relations and on noting that for any $i \in \mathbf{n} \backslash \mathbf{K}$ by definition $\theta_{\Pi_{i}}^{p_{i}}=\theta_{\Pi_{i}}^{p_{i}}$, one arrives at the following inequality:

$$
\dot{\theta}<\sum_{i=1}^{n} \theta_{\Pi_{i}}^{p_{i}}
$$

Now, it results from (6) and (9) that:

$$
\dot{\theta}<\theta
$$

which implies that using the sensor relocation scheme intro- 
duced in this work the total weighted coverage increases.

The proposed deployment strategies will be presented in the sequel.

\section{A. The Maximum Weighted Vertex (MWV) Strategy}

In this strategy, each sensor moves toward the vertex with maximum weight in its MW-Voronoi region. This vertex is referred to as the heaviest vertex, and is denoted by $V_{i, \max }$ for the $i$-th region. According to this strategy, all sensors search for any coverage holes in their MW-Voronoi regions. Once the coverage holes are detected, each sensor identifies the heaviest vertex in its MW-Voronoi region. Then, for any $i \in \mathbf{n}, S_{i}$ moves toward $V_{i, \max }$ and continues moving until it is covered. This occurs when the distance of the $i$-th sensor from $V_{i, \max }$ is equal to its sensing radius.

As an operational example of the MWV strategy, consider 27 sensors randomly deployed in a $50 \mathrm{~m} \times 50 \mathrm{~m}$ flat space: 15 with a sensing radius of $1 \mathrm{~m}, 6$ with a sensing radius of $\frac{5}{6} \mathrm{~m}$, 3 with a sensing radius of $\frac{7}{6} \mathrm{~m}$, and 3 with a sensing radius of $1.5 \mathrm{~m}$. Moreover, the communication range of each sensor is assumed to be $20 \mathrm{~m}$. The priority function representing the network coverage priority in this example is given by $\varphi(q)=\exp \left(-0.4\left[\left(x_{q}-25\right)^{2}+\left(y_{q}-25\right)^{2}\right]\right)$, where $x_{q}$ and $y_{q}$ are the abscissa and ordinate of the point $q$, respectively. Each point in the field is represented by a gray level proportional to the coverage priority of that point. In Fig. 3, three snapshots are provided, and in each one both the sensing circles of every sensor (filled circles) and the MW-Voronoi regions are depicted. It can be observed from this figure that in the final round the sensors concentrate on the area with higher coverage priority.

While the sensor deployment strategy discussed above proves effective in many practical cases, it may not be as effective when there are small number of sensors with small sensing ranges in the field. In such cases, the MW-Voronoi regions are relatively large, and hence there is a good chance that each area with high coverage priority is mainly located in only one of the regions (see Fig. 4). Thus, by moving toward the heaviest vertex in a region, the high-priority area in that region might not be covered. For example, under the MWV strategy the sensor $S_{4}$ in Fig. 4 would move toward the vertex $V_{4, \max }$, missing the high-priority area which is roughly in the opposite direction. Furthermore, in the case when the priority function varies significantly over some regions, the corresponding sensors might not move in the proper direction. In the special case, if an MW-Voronoi region has no vertices (i.e., it is a circle), the corresponding sensor does not move under the MWV strategy, which is another shortcoming of this strategy. To remedy the above-mentioned problems, a new deployment technique will be presented in the next subsection.

\section{B. The Maximum Weighted Point (MWP) strategy}

In this strategy, each sensor moves to a point in its MWVoronoi region which has the maximum weight. This point will be referred to as the heaviest point, and is denoted by $p_{i, \max }$ for the $i$-th region. According to this strategy, once a

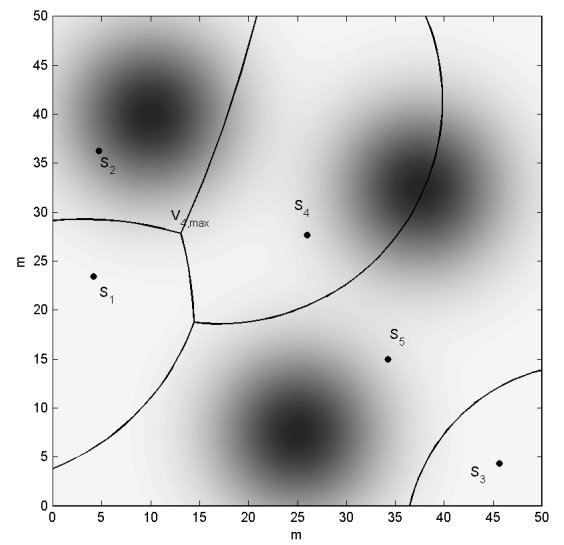

Fig. 4: A network of 5 mobile sensors in a weighted field, where the MWV algorithm is not as effective because the sensing range of every sensor is small.

coverage hole is discovered in an MW-Voronoi region, the corresponding sensor finds the heaviest point in that region and moves toward it up to the position from which $p_{i, \max }$ is covered.

As an example, consider the initial deployment of Fig. 5, and let the priority function be equal to $\varphi(q)=\exp \left(-0.4\left[\left(x_{q}-\right.\right.\right.$ $\left.\left.10)^{2}+\left(y_{q}-40\right)^{2}\right]\right)+\exp \left(-0.4\left[\left(x_{q}-25\right)^{2}+\left(y_{q}-7.5\right)^{2}\right]\right)+$ $\exp \left(-0.4\left[\left(x_{q}-37.5\right)^{2}+\left(y_{q}-32.5\right)^{2}\right]\right)$. Let also 18 sensors with a communication range of $20 \mathrm{~m}$ be randomly deployed in the field: 10 with a sensing radius of $2 \mathrm{~m}, 4$ with a sensing radius of $\frac{5}{3} \mathrm{~m}, 2$ with a sensing radius of $\frac{7}{3} \mathrm{~m}$, and 2 with a sensing radius of $3 \mathrm{~m}$. As it can be observed from Fig. 5, after the final round the sensors are more concentrated on highpriority areas in the field.

\section{The Maximum Distance Weight (MDW) Strategy}

The two weight-based techniques discussed thus far are not suitable when the priority function is smooth. For instance, when the weight of all points of the field are equal (i.e., $\varphi(q)=$ constant), sensors do not move under the MWV and MWP strategies. This motivates the development of a new strategy called MDW, which operates based on both distance and weight.

For any $i \in \mathbf{n}$, the MDW strategy finds a point inside the $i$-th MW-Voronoi region whose distance from $S_{i}$ multiplied by its weight is maximum. This point will be referred to as the $i$-th $M D W$ centroid, and will be denoted by $p_{i, M D W}$. Once this point is obtained, $S_{i}$ moves toward it and continues moving until $p_{i, M D W}$ is covered. This occurs when the distance of the $i$-th sensor from the point $p_{i, M D W}$ is equal to its sensing radius.

Fig. 6 shows an operational example of the MDW strategy. In this example, 27 sensors with the communication range of $20 \mathrm{~m}$ are randomly deployed in a $50 \mathrm{~m}$ by $50 \mathrm{~m}$ flat space: 15 with a sensing radius of $3 \mathrm{~m}, 6$ with a sensing radius of $2.5 \mathrm{~m}, 3$ with a sensing radius of $3.5 \mathrm{~m}$, and 3 with a sensing radius of $4.5 \mathrm{~m}$. The priority function for this example is $\varphi(q)=\exp \left(-0.004\left[\left(x_{q}-25\right)^{2}+\left(y_{q}-25\right)^{2}\right]\right)$. It can be observed from this figure that in the final round the weighted coverage significantly increases. 


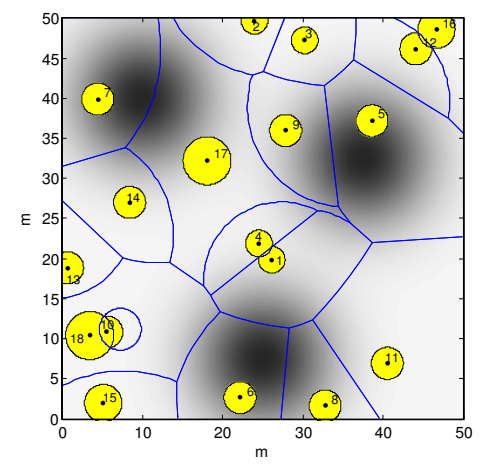

(a)

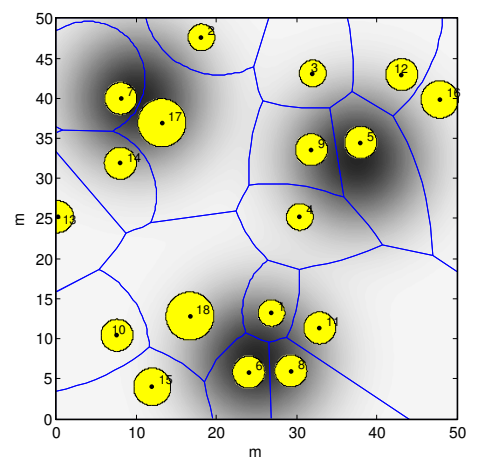

(b)

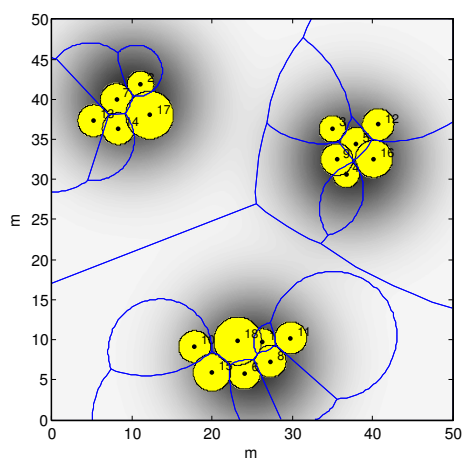

(c)

Fig. 5: Snapshots of the execution of the MWP strategy where different gray levels are used to indicate the coverage priorities, similar to Fig. 3. (a) Initial coverage; (b) coverage after the first round, and (c) final coverage.

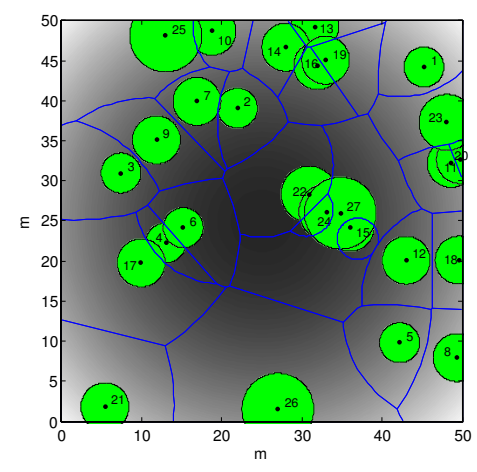

(a)

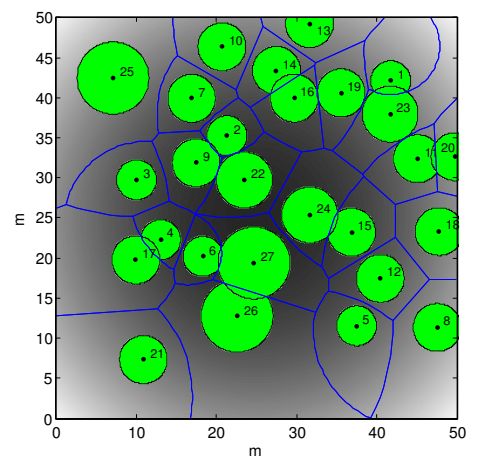

(b)

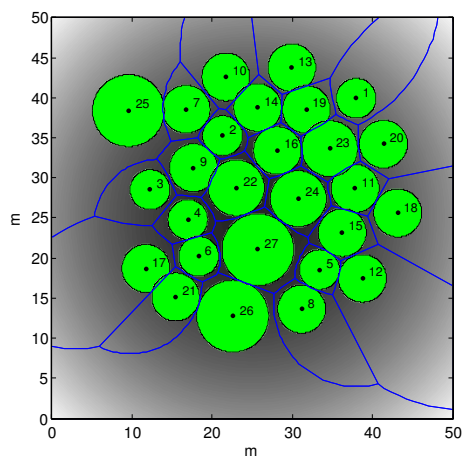

(c)

Fig. 6: Snapshots of the execution of the MDW strategy where different gray levels are used to indicate the coverage priorities, similar to Fig. 3. (a) Initial coverage; (b) coverage after the first round, and (c) final coverage.

Remark 2. It is worth mentioning that for the case when all sensors have the same sensing capability and the weight of every point in the field is the same, the MDW strategy will be the same as the VOR strategy proposed in [7]. In other words, the MDW strategy proposed here is the generalized form of the VOR technique.

Remark 3. In order to prevent sensors from oscillatory movements, a control mechanism similar to the one in [7] can be used here. Under this mechanism, each sensor compares the newly computed direction with the previous one, and will move only if the new direction is consistent with that in the preceding round.

Remark 4. It is important to note that even in the case of a centralized deployment scheme, no optimal solution is available, in general, for the coverage problem in a nonuniform sensing field.

Remark 5. One of the advantages of using the MW-Voronoi diagram for nonidentical sensors is that it guarantees the con- vergence of the sensor trajectories. Note that the monotonically increasing behavior of total weighted coverage (shown in Theorem 1) yields only with the MW-Voronoi partitioning and not with the conventional Voronoi partitioning. More precisely, relation (6) in the proof of Theorem 1 does not hold if the conventional Voronoi diagram is used to partition the field in the presence of nonidentical sensors. This means that using existing sensor deployment strategies (which assume identical sensing capability for sensors) may lead to non-convergent sensor movements.

\section{Comparative Study}

In this section, the three algorithms proposed in Section IV are applied to a flat space of size $50 \mathrm{~m} \times 50 \mathrm{~m}$ with different number of sensors. In these simulations, the algorithms terminate when none of the sensors' weighted coverage in its corresponding MW-Voronoi region would increase by more than $1 \%$ in the next move. Note that the results presented in this section for weighted coverage are all the average values obtained by using 20 random initial deployments for sensors. 
Remark 6. The sensing field is divided into small grid cells, and some computations are performed over the centers of the cells, which will be referred to as the grid centers in the sequel. The choice of the cell size is made based on the distribution of the priority function in different points of the field, desired precision, and processing capability of different sensors. The computational task for each algorithm consists of three phases, which are performed by each sensor individually. In the first phase, every sensor finds those grid centers which lie inside its MW-Voronoi region (this is done by checking the algebraic condition (2) for all grid centers). The second phase depends on the particular strategy adopted: In the MWV method each sensor finds the heaviest vertex in its MW-Voronoi region (note that each vertex is the intersection of two Apollonian circles). In the MWP and MDW techniques, on the other hand, each sensor finds a proper point among all grid centers in its MWVoronoi region. In the third phase, the local weighted coverage of every sensor is compared with its weighted coverage w.r.t. the newly computed destination point. To this end, the weights of the corresponding grid centers in each MW-Voronoi region are summed up and the result is multiplied by the area of each cell. It is worth mentioning that with the current stateof-the-art technology for industrial sensors (e.g. Mica2 [27] or Epic [28]), the computations described above can be efficiently carried out in a short period of time.

Example 1. In this example, 27 sensors with a communication range of $20 \mathrm{~m}$ are randomly deployed in the flat space described above: 15 with a sensing radius of $6 \mathrm{~m}, 6$ with a sensing radius of $5 \mathrm{~m}, 3$ with a sensing radius of $7 \mathrm{~m}$, and 3 with a sensing radius of $9 \mathrm{~m}$. The priority function representing the importance of coverage of different points in the field is assumed to be $\varphi(q)=\exp \left(-0.4\left[\left(x_{q}-25\right)^{2}+\left(y_{q}-25\right)^{2}\right]\right)$ for this example. Fig. 7 depicts the weighted coverage factor, defined as the ratio of the total weighted coverage to the total field weight, after each round of the three algorithms. The figure shows that the performances of the MDW and $M W V$ strategies in this example are more or less the same. In fact, it can be verified that when a relatively large number of sensors with large sensing ranges are distributed in the field, all three algorithms reach a satisfactory weighted coverage. Since the computational complexity for finding the point $V_{i, \max }$ is less than that for finding $p_{i, \max }$ and $p_{i, M D W}$, the $M W V$ algorithm is more efficient in such scenarios as far as the processing capability of the sensors is concerned.

Example 2. Consider 9 sensors with the communication range of $20 \mathrm{~m}$ randomly deployed in the flat space described earlier: 5 with a sensing radius of $1 m, 2$ with a sensing radius of $\frac{5}{6} \mathrm{~m}, 1$ with a sensing radius of $\frac{7}{6} \mathrm{~m}$, and 1 with a sensing radius of $1.5 \mathrm{~m}$. Let the priority function be equal to $\varphi(q)=$ $\exp \left(-k\left[\left(x_{q}-10\right)^{2}+\left(y_{q}-40\right)^{2}\right]\right)+\exp \left(-k\left[\left(x_{q}-25\right)^{2}+\left(y_{q}-\right.\right.\right.$ $\left.\left.7.5)^{2}\right]\right)+\exp \left(-k\left[\left(x_{q}-37.5\right)^{2}+\left(y_{q}-32.5\right)^{2}\right]\right)$, where $k=0.4$. Due to the relatively small number of sensors in this example (compared to the field size), the MW-Voronoi regions are comparatively large. Furthermore, since the priority function

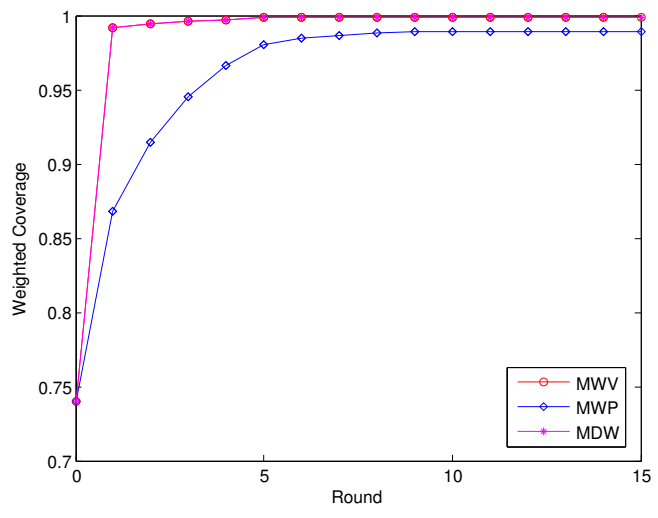

Fig. 7: The weighted coverage per round for Example 1.

is sharp (concentrated in three different areas), each area with a large weight will likely lie mainly inside one $M W$-Voronoi region (not on its boundaries). On the other hand, because of the relatively small sensing radius of the sensors, there is a good chance that these important areas would not be covered by moving toward the vertex with maximum weight in the MWV strategy or toward the point with the maximum weighted distance from the corresponding sensor in the $M D W$ strategy. Hence, the MWP algorithm outperforms the other two in this case (see Fig. 8).

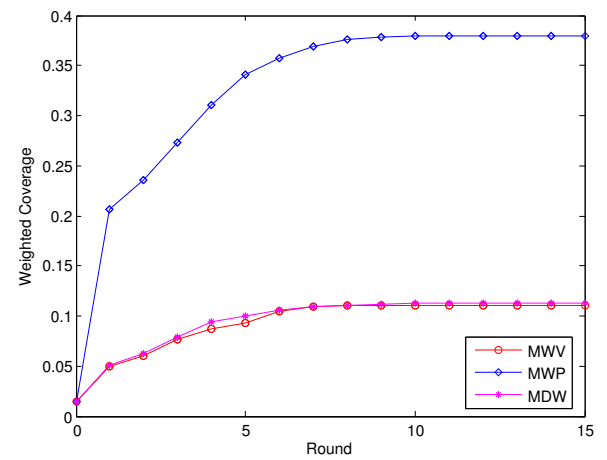

Fig. 8: The weighted coverage per round for Example 2.

Example 3. Consider the sensor network described in Example 1 , and let the priority function here be of the same form as in the previous example, but with $k=0.004$. This priority function is relatively smooth, and confirmed by Fig. 9, the MWV and MWP strategies are not as effective as the $M D W$ strategy for this case. In addition, since the sensing ranges of the sensors are relatively large, it is more likely that the sensors will have overlapped sensing areas, if they move to the heaviest points or vertices, without taking the traveling distance into consideration. In general, when the priority function is not sharp and the sensing ranges of the sensors are relatively large, the MDW strategy outperforms the other two.

Example 4. The performance of the proposed algorithms is 


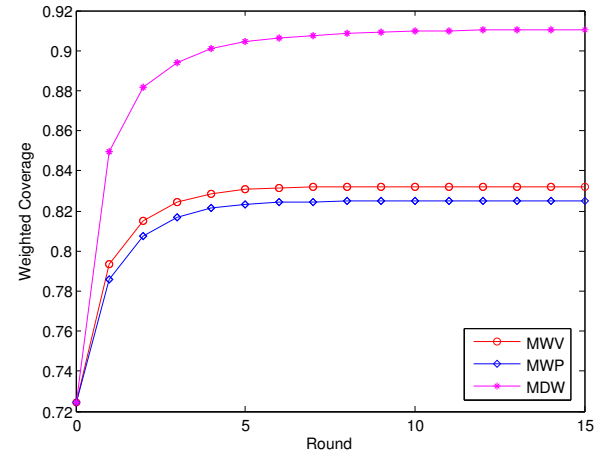

Fig. 9: The weighted coverage per round for Example 3.

investigated for two different setups in this example. The first setup is the same as the one in Example 2, and the second setup is the same as that in Example 1. In both scenarios, it is assumed that initially the sensors are distributed randomly in the field. The priority function is of the same form as in Example 2 in both setups. Fig. 10 depicts the final weighted coverage for different values of $k$, in the first setup. As it can be observed from this figure, when there are a small number of sensors with small sensing ranges in the field, the MWP strategy results in a better weighted coverage compared to the other two algorithms, and this superiority is considerable when the priority function is sharp. Fig. 11 shows the final weighted coverage for different values of $k$ in the second setup. As it can be seen from this figure, when a large number of sensors with high sensing capabilities are distributed in the field, the MDW strategy outperforms the other two. As mentioned before, this superiority is significant when the priority function is smooth.

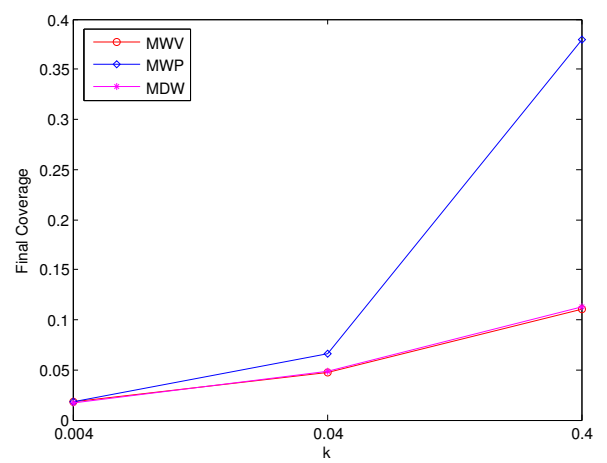

Fig. 10: The final weighted coverage for different values of $k$, in the first scenario of Example 4.

Energy efficiency is an important issue in the design of sensor deployment strategies, and should be taken into consideration in the performance evaluation. The energy consumption of a mobile sensor is mainly due to movement, and highly depends on the traveling distance of the sensor as well as the number of times it stops before arriving at the desired position (the latter is due to the static friction). Let the required energy for traveling $1 \mathrm{~m}$ (without stopping) be $8.268 \mathrm{~J}$

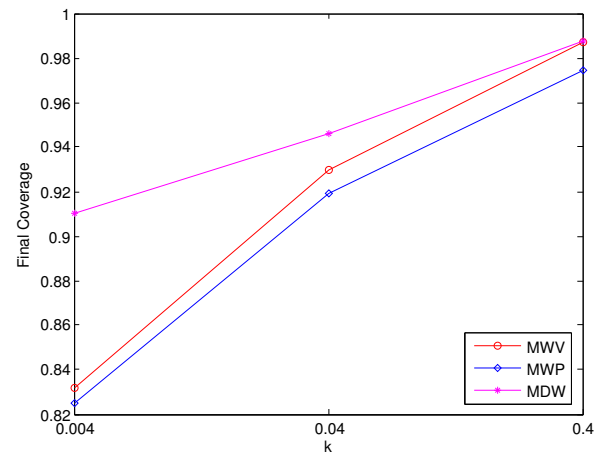

Fig. 11: The final weighted coverage for different values of $k$, in the second scenario of Example 4.

(or $0.210 \mathrm{~J} / \mathrm{inch}$ ) [29], [30]. Assume that the energy required to stop a sensor and then overcome its static friction after a complete stop is equal to the energy required to continuously move the sensor one meter [7], [13]. Table I provides the average energy consumption for Examples 1-4.

Another important means of assessing the performance of sensor deployment strategies is the time it takes to reach the desired termination condition. Given that the sensor deployment time in each round is more or less the same for all strategies, the number of rounds required to meet a predetermined termination criterion can be used as a measure of the deployment speed. Table II shows the stopping round for the four examples given above. Similar to Table I, here also the entries of the table are the average values obtained by running 20 simulations with different initial conditions (this explains why the number of rounds in Table II are non-integer).

Example 5. Three operational scenarios are considered here for the special case of identical sensors. The communication range of the sensors in all three scenarios is $20 \mathrm{~m}$, and initially they are assumed to be placed randomly in the field described earlier. In the first scenario, 30 sensors with a sensing range of $1 \mathrm{~m}$ are considered, and the priority function is the same as that in Example 1. Three snapshots in this case are provided in Fig. 12, and in each one the sensing circles of every sensor (filled circles) as well as the Voronoi polygons are depicted. It can be observed from this figure that in the final round the sensors are more concentrated in the area with higher coverage priority.

In the second scenario, 15 sensors with a sensing range of $2 m$ are deployed in the field, with the same priority function as in Example 2. Three snapshots are provided in Fig. 13, similar to Fig. 12, which show the good performance of the MWP strategy for this scenario.

In the third scenario, 30 sensors with a sensing range of $3 m$ are considered, and the priority function is $\varphi(q)=$ $\exp \left(-0.004\left[\left(x_{q}-25\right)^{2}+\left(y_{q}-25\right)^{2}\right]\right)$ as shown in Fig. 14. It can be observed from this figure that in the final round the total weighted coverage significantly increases.

It is to be noted that in all three scenarios presented above the resultant Voronoi regions are polygons as the sensing radii 
TABLE I: The energy consumption (in Joule) for Examples 1-4 using the proposed algorithms.

\begin{tabular}{|c|c|c|c|c|c|c|c|c|c|}
\hline & Example 1 & Example 2 & Example 3 & $\begin{array}{c}\text { Example 4 } \\
K=0.004 \\
\text { First Scenario }\end{array}$ & $\begin{array}{c}\text { Example 4 } \\
K=0.04 \\
\text { First Scenario }\end{array}$ & $\begin{array}{c}\text { Example 4 } \\
K=0.4 \\
\text { First Scenario }\end{array}$ & $\begin{array}{c}\text { Example 4 } \\
K=0.004 \\
\text { Second Scenario }\end{array}$ & $\begin{array}{c}\text { Example 4 } \\
K=0.04 \\
\text { Second Scenario }\end{array}$ & $\begin{array}{c}\text { Example 4 } \\
K=0.4 \\
\text { Second Scenario }\end{array}$ \\
\hline MWV & 35.78 & 115.13 & 28.09 & 113.03 & 145.99 & 115.13 & 28.09 & 31.00 & 29.12 \\
\hline MWP & 33.83 & 129.84 & 26.36 & 104.53 & 118.11 & 129.84 & 26.36 & 26.93 & 25.76 \\
\hline MDW & 36.04 & 118.42 & 47.70 & 100.82 & 167.52 & 118.42 & 47.70 & 34.35 & 29.57 \\
\hline
\end{tabular}

TABLE II: The number of rounds required for different algorithms to terminate in Examples 1-4.

\begin{tabular}{|c|c|c|c|c|c|c|c|c|c|}
\hline & Example 1 & Example 2 & Example 3 & $\begin{array}{c}\text { Example 4 } \\
K=0.004 \\
\text { First Scenario }\end{array}$ & $\begin{array}{c}\text { Example 4 } \\
K=0.04 \\
\text { First Scenario }\end{array}$ & $\begin{array}{c}\text { Example 4 } \\
K=0.4 \\
\text { First Scenario }\end{array}$ & $\begin{array}{c}\text { Example 4 } \\
K=0.004 \\
\text { Second Scenario }\end{array}$ & $\begin{array}{c}\text { Example 4 } \\
K=0.04 \\
\text { Second Scenario }\end{array}$ & $\begin{array}{c}\text { Example 4 } \\
K=0.4 \\
\text { Second Scenario }\end{array}$ \\
\hline MWV & 8.25 & 6.95 & 7.25 & 4.95 & 7.2 & 6.95 & 7.25 & 8.75 & 7.30 \\
\hline MWP & 9.30 & 8.65 & 8.25 & 4.60 & 7.2 & 8.65 & 8.25 & 8.65 & 8.45 \\
\hline MDW & 8.00 & 7.15 & 8.85 & 2.85 & 6.00 & 7.15 & 8.85 & 8.30 & 7.35 \\
\hline
\end{tabular}

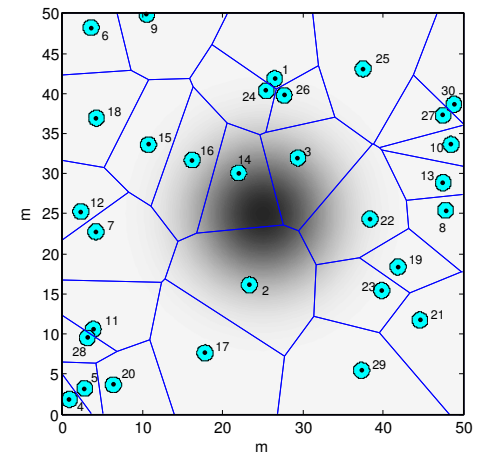

(a)

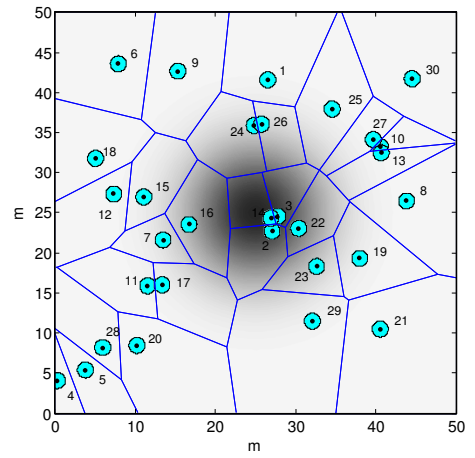

(b)

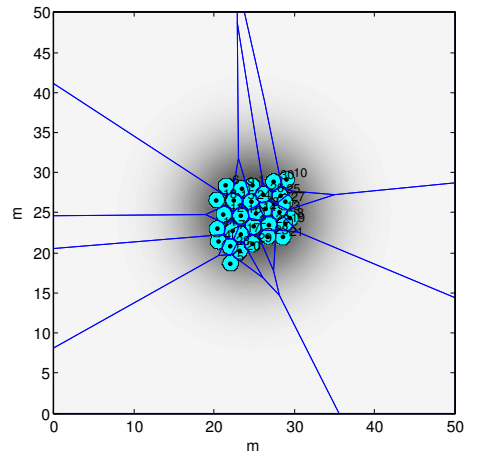

(c)

Fig. 12: Snapshots of the execution of the MWV strategy for the first scenario of Example 5 (the coverage priority is indicated by different gray levels). (a) Initial coverage; (b) coverage after the first round, and (c) final coverage.

of all sensors are the same.

Example 6. The performance of the proposed algorithms is now compared with the Minmax-point algorithm [8] which is an effective coverage strategy for nonidentical sensors with uniform sensing priority. In this example, 27 sensors with a communication range of $20 \mathrm{~m}$ are randomly deployed in the flat space described earlier: 15 with a sensing radius of $3 m$, 6 with a sensing radius of $2.5 \mathrm{~m}, 3$ with a sensing radius of $3.5 \mathrm{~m}$, and 3 with a sensing radius of $4.5 \mathrm{~m}$. Let the priority function be equal to $\varphi(q)=\exp \left(-k\left[\left(x_{q}-25\right)^{2}+\left(y_{q}-25\right)^{2}\right]\right.$; the smaller $k$ is the closer the sensing priority is to being uniform. In the special case, when $k=0$ the sensing priority throughout the field is uniform. Fig. 15 shows the final weighted coverage for four different values of $k: k=0,0.001,0.01,0.1$. As it can be seen from this figure, the proposed algorithms outperform the Minmax-point algorithm when the target field is non-uniform (i.e., $k \neq 0$ ) and this superiority is more significant for a more non-uniform priority function. Note that when the target field is uniform, the sensors do not move under the MWV and MWP algorithms, and in this case the Minmax-point algorithm is more effective than these two. However, even in this case the performance of the MDW algorithm is better than that of the Minmax-point algorithm.

Remark 7. The overall performance of a coverage strategy highly depends on the specific application and network configuration in terms of the number of sensors, priority function, sensing range of the sensors and computational power of the mobile agents. In order to select the proper coverage strategy (which is done by the operator in the beginning), a number of issues should be taken into account. For example, as far as computational complexity is concerned, the MWV deployment strategy outperforms the other two techniques. On the other hand, when the priority function is more or less the same over the entire field the MDW strategy is more effective. If the priority function is highly non-uniform (e.g., it is very much focused in certain areas), then the proper choice of algorithm 


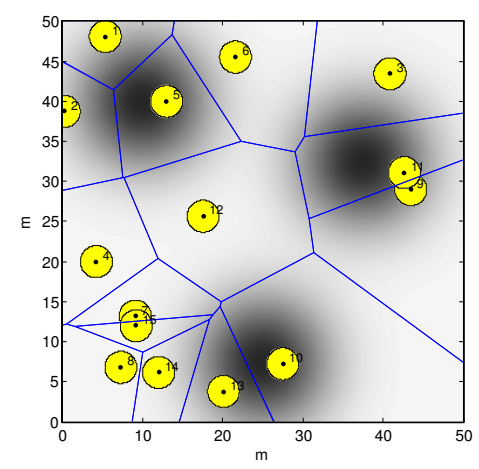

(a)

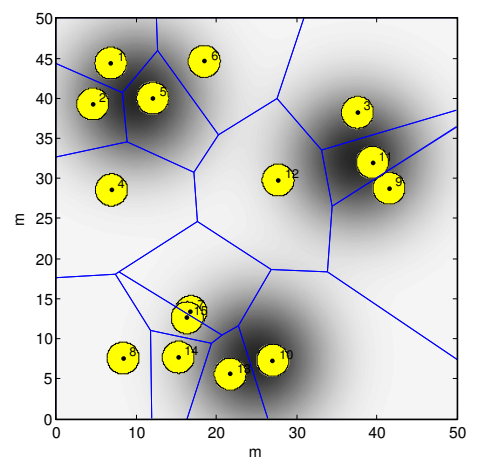

(b)

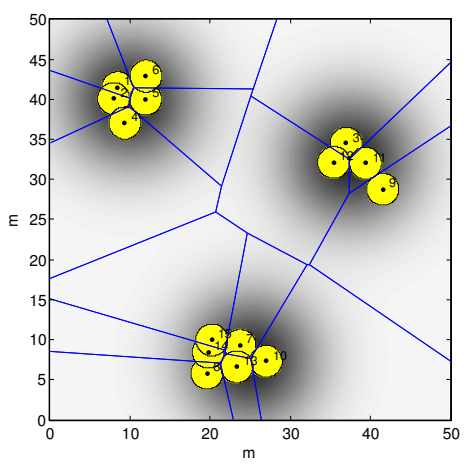

(c)

Fig. 13: Snapshots of the execution of the MWP strategy for the second scenario of Example 5 (the coverage priority is indicated by different gray levels). (a) Initial coverage; (b) coverage after the first round, and (c) final coverage.

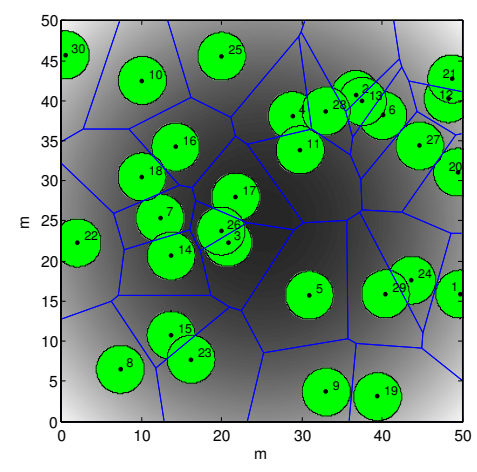

(a)

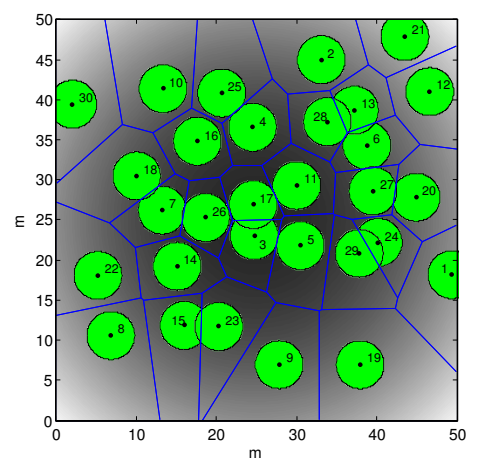

(b)

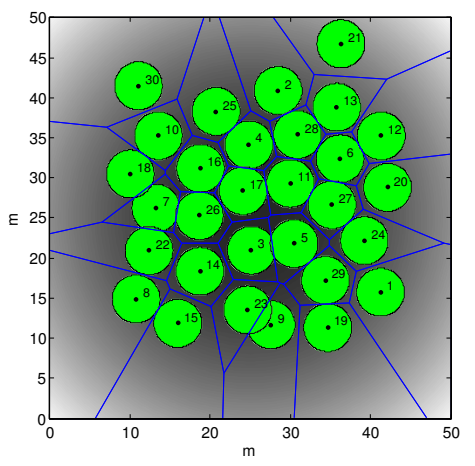

(c)

Fig. 14: Snapshots of the execution of the MDW strategy for the third scenario of Example 5 (the coverage priority is indicated by different gray levels). (a) Initial coverage; (b) coverage after the first round, and (c) final coverage.

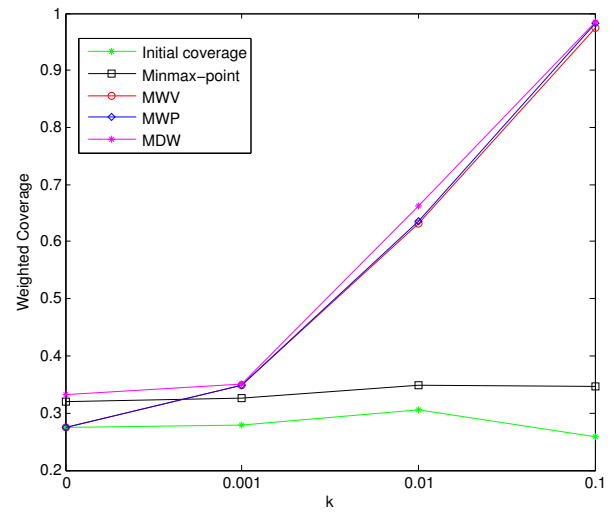

Fig. 15: The weighted coverage for different values of $k$ in Example 6.

depends on the number of sensors and their sensing radii. Particularly, for a small number of sensors with small sensing radii and a highly non-uniform priority function, the MWP strategy outperforms the other two techniques.

\section{CONCLUSIONS}

Efficient sensor deployment algorithms are presented to increase coverage in a mobile sensor network with a prescribed priority assignment for different points in the sensing field. It is assumed that the sensors are not identical in terms of sensing capabilities. The multiplicatively weighted Voronoi (MW-Voronoi) diagram is then employed to develop three distributed deployment strategies. According to the proposed algorithms, each sensor moves iteratively in such a way that the prioritized uncovered area in its MW-Voronoi region is reduced. All proposed algorithms consider the relative priority of the points inside each region (or on its vertices). One of these strategies also takes the distances of each sensor and the points inside its MW-Voronoi region into account. Simulations are presented to compare the performance of the coverage algorithms developed in this work. 


\section{ACKNOWLEDGMENT}

The authors would like to thank Dr. Kaveh Moezzi and Mr. Walid Masoudimansour for helpful discussions and suggestions.

\section{REFERENCES}

[1] C. Intanagonwiwat, R. Govindan, and D. Estrin, "Directed diffusion: A scalable and robust communication paradigm for sensor networks," in Proceedings of 6th Annual International Conference on Mobile Computing and Networking, 2000, pp. 56-67.

[2] G. J. Pottie and W. J. Kaiser, Wireless Integrated Network Sensors. ACM New York, NY, USA, 2000.

[3] H. Mahboubi, A. Momeni, A. G. Aghdam, K. Sayrafian-Pour, and V. Marbukh, "An efficient target monitoring scheme with controlled node mobility for sensor networks," IEEE Transactions on Control Systems Technology, vol. 20, no. 6, pp. 1522-1532, 2012.

[4] T. Clouqueur, V. Phipatanasuphorn, P. Ramanathan, and K. K. Saluja, "Sensor deployment strategy for target detection," in Proceedings of 1st ACM International Workshop on Wireless Sensor Networks and Applications, 2002, pp. 42-48.

[5] S. Meguerdichian, F. Koushanfar, M. Potkonjak, and M. B. Srivastava, "Coverage problems in wireless ad hoc sensor networks," in Proceedings of IEEE INFOCOM, 2001, pp. 1380-1387.

[6] A. Howard, M. J. Matarić, and G. S. Sukhatme, "An incremental selfdeployment algorithm for mobile sensor networks," Autonomous Robots, vol. 13, no. 2, pp. 113-126, 2002.

[7] G. Wang, G. Cao, and T. F. L. Porta, "Movement-assisted sensor deployment," IEEE Transactions on Mobile Computing, vol. 5, no. 6, pp. 640-652, 2006.

[8] H. Mahboubi, K. Moezzi, A. G. Aghdam, K. Sayrafian-Pour, and V. Marbukh, "Self-deployment algorithms for coverage problem in a network of mobile sensors with unidentical sensing range," in Proceedings of IEEE Global Communications Conference, 2010, pp. 1-6.

[9] H. Mahboubi, K. Moezzi, A. G. Aghdam, and K. Sayrafian-Pour, "Selfdeployment algorithms for field coverage in a network of nonidentical mobile sensors," in Proceedings of IEEE International Conference on Communications, 2011, pp. 1-6.

[10] T. Clouqueur, V. Phipatanasuphorn, P. Ramanathan, and K. K. Saluja, "Sensor deployment strategy for detection of targets traversing a region," ACM Mobile Networks and Applications, vol. 8, pp. 453-461.

[11] J. Luo and Q. Zhang, "Probabilistic coverage map for mobile sensor networks," in Proceedings of IEEE Global Communications Conference, 2008, pp. 357-361.

[12] A. Konstantinidis, K. Yang, and Q. Zhang, "An evolutionary algorithm to a multi-objective deployment and power assignment problem in wireless sensor networks," in Proceedings of IEEE Global Communications Conference, 2008, pp. 475-480.

[13] G. Wang, G. Cao, P. Berman, and T. F. L. Porta, "A bidding protocol for deploying mobile sensors," IEEE Transactions on Mobile Computing, vol. 6, no. 5, pp. 563-576, 2007.

[14] A. Kwok and S. Martinez, "Unicycle coverage control via hybrid modeling," IEEE Transactions on Automatic Control, vol. 55, no. 2, pp. 528-532, 2010.

[15] J. Cortes, S. Martinez, and F. Bullo, "Spatially-distributed coverage optimization and control with limited-range interactions," ESAIM. Control, Optimization and Calculus of Variations, vol. 11, pp. 691-719, 2005.

[16] F. Bullo, J. Cortes, and S. Martinez, Distributed Control of Robotic Networks. Princeton University Press, 2009.

[17] J. Cortes, "Coverage optimization and spatial load balancing by robotic sensor networks," IEEE Transactions on Automatic Control, vol. 55, no. 3, pp. 749-754, 2010.

[18] E. Deza and M. M. Deza, Encyclopedia of Distances. Springer, 2009.

[19] A. Okabe, B. Boots, K. Sugihara, and S. N. Chiu, Spatial Tessellations: Concepts and Applications of Voronoi Diagrams. Wiley, 2000.

[20] R. Reitsma, S. Trubin, and E. Mortensen, "Weight-proportional space partitioning using adaptive voronoi diagrams," Geoinformatica, vol. 11, pp. 383-405, 2007.

[21] R. Klein, Concrete and Abstract Voronoi Diagrams. Springer, 1989.

[22] A. V. Akopyan and A. A. Zaslavsky, Geometry of Conics. American Mathematical Society, 2007.

[23] D. E. Koditschek, Robot Planning and Control via Potential Functions. MIT Press Cambridge, MA, USA, 1989.
[24] Q. Li, M. D. Rosa, and D. Rus, "Distributed algorithms for guiding navigation across a sensor network," in Proceedings of 9th Annual International Conference on Mobile Computing and Networking, 2003, pp. 313-325.

[25] D. Niculescu and B. Nath, "Ad hoc positioning system (aps) using aoa," in Proceedings of IEEE INFOCOM. Twenty-Second Annual Joint Conference of the IEEE Computer and Communications Societies, 2003, pp. 1734-1743.

[26] C. Rentel and T. Kunz, "A mutual network synchronization method for wireless ad hoc and sensor networks," IEEE Transactions on Mobile Computing, vol. 7, pp. 633-646, 2008.

[27] P. Ballal and F. Lewis, "Introduction to Crossbow Mica2 Sensors," Course Note, University of Texas at Arlington, 2007.

[28] P. Dutta, J. Taneja, J. Jeong, X. Jiang, and D. Culler, "A building block approach to sensornet systems," in Proceedings of 6th ACM Conference on Embedded Network Sensor Systems, 2008.

[29] S. Yoon, O. Soysal, M. Demirbas, and C. Qiao, "Coordinated locomotion and monitoring using autonomous mobile sensor nodes," IEEE Transactions on Parallel and Distributed Systems, vol. 22, no. 10, pp. 1742-1756, 2011.

[30] M. Rahimi, H. Shah, G. S. Sukhatme, J. Heideman, and D. Estrin, "Studying the feasibility of energy harvesting in a mobile sensor network," in Proceedings of IEEE International Conference on Robotics and Automation, vol. 1, 2003, pp. 19-24.

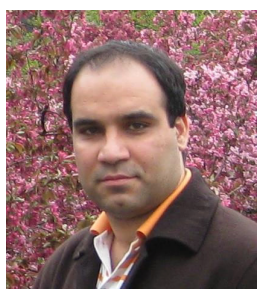

Hamid Mahboubi is a recipient of the Gold Medal in the 1999 National Math Olympiad in Iran. He was awarded an honorary admission to Sharif University of Technology, Tehran, Iran, where he received his B.Sc. degree in Electrical Engineering in 2003. He received his M.A.Sc. degree in Electrical and Computer Engineering from the University of Tehran, Tehran, Iran, in 2006. He is currently a Ph.D. candidate in the Department of Electrical and Computer Engineering at Concordia University, Montreal, Canada. Mr. Mahboubi is the recipient of Bourse d'Etudes Hydro Quebec Scholarship, Power Corporation of Canada Graduate Fellowship, Canadian National Award in Transportation, and Concordia University Graduate Fellowship. He has served as Chair of the Control Systems Chapter of the IEEE Montreal Section since January 2012. His research interests include mobile sensor networks, multi-agent systems, hybrid systems and optimization.

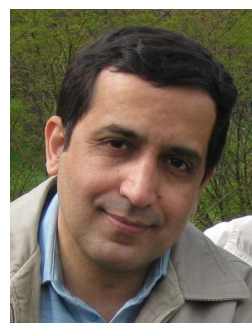

Jalal Habibi received the B.A.Sc. and M.A.Sc. degrees in Electrical Engineering from Sharif University of Technology, Tehran, Iran in 1997 and 2000, respectively and the Ph.D. degree from the University of Tehran, Tehran, Iran in 2008. He was an Assistant Professor with the Science and Research Branch of Azad University, Tehran, from 2009 to 2010. He joined Concordia University, Montreal, Canada as a Postdoctoral Fellow in 2010, where he is currently a Research Associate with the Department of Electrical and Computer Engineering.

His research interests include predictive control, hybrid systems, mathematical optimization and sensor networks. Dr. Habibi is an Associate Editor of the Canadian Journal of Electrical and Computer Engineering and a member of IEEE. 


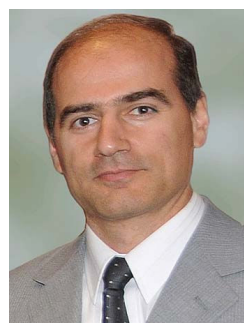

Amir G. Aghdam received the B.A.Sc. degree from Isfahan University of Technology, Isfahan, Iran and the M.A.Sc. degree from Sharif University of Technology, Tehran, Iran, both in Electrical Engineering. He received his Ph.D. degree from the University of Toronto in 2000 and worked as a development engineer at Voyan Technology, Santa Clara, California from 2000 to 2001. He was a postdoctoral researcher at the systems control group of the University of Toronto for six months and then joined Concordia University in 2002, where he is currently an Associate Professor in the Department of Electrical and Computer Engineering. Dr. Aghdam is a member of Professional Engineers of Ontario and a senior member of the IEEE. He served as Chair of the IEEE Montreal Section and Chair of the Control Systems Chapter of the IEEE Montreal Section in 2005 and 2006, and Chair of the IEEE Eastern Canada Area in 2007 to 2009. He was the Editor-in-Chief of IEEE Canadian Review (20102012), and General Chair of the 2012 IEEE Canadian Conference on Electrical and Computer Engineering. Dr. Aghdam is a Regional Editor of the IEEE Systems Journal, an Associate Editor of the IEEE Transactions on Control Systems Technology, and has been a Technical Program Committee Member of several conferences including IEEE Conference on Control Applications, IEEE Conference on Decision and Control, and American Control Conference. $\mathrm{He}$ is a recipient of the 2009 IEEE MGA Achievement Award, and 2011 IEEE Canada J. J. Archambault Eastern Canada Merit Award. His research interests include decentralized control of large-scale systems, multi-agent systems, switching control, and sampled-data systems.

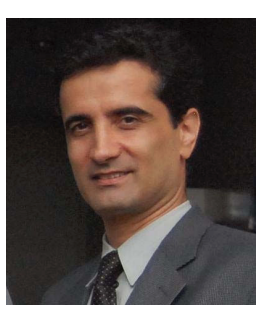

Kamran Sayrafian-Pour holds Ph.D., M.S. and B.S. degrees in Electrical \& Computer Engineering from University of Maryland, Villanova University and Sharif University of Technology, respectively. $\mathrm{He}$ is currently a program lead at the Information Technology Laboratory of the National Institute of Standards and Technology (NIST) located in Gaithersburg, Maryland. Prior to this, he was the cofounder of Zagros Networks, Inc. a fabless semiconductor company based in Rockville, Maryland where he served as President and senior member of the architecture team. Dr. Sayrafian-Pour has been an adjunct faculty of the University of Maryland since 2003. He has served as an invited member of the technical program committee of many international conferences. He has also been the co-chair and organizer of several IEEE ComSoc Conference tracks and workshops focused on application of wireless communication in healthcare. His research interests include medical body area networks, mobile sensor networks and RF-based indoor positioning. He has published over 70 conference and journal papers, and book chapters in these areas. He was the recipient of the IEEE PIMRC 2009 \& SENSORCOMM 2011 best paper awards. He has been a Guest Editor for a number of special issues focusing on the pervasive healthcare technologies and sensor networks. Dr. Sayrafian-Pour is a member of the COST Action IC1004 Cooperative Radio Communications for Green Smart Environments; and, his research results have been included in the final report of the COST Action 2100 (Pervasive Mobile and Ambient Wireless Communications), which was published by Springer in January 2012 He was also a contributing member and the co-editor of the channel modeling document of the IEEE802.15.6 international standardization on body area networks. He is the co-inventor/inventor of four U.S. patents and a senior member of IEEE. 